\title{
Monitoring System Design and Network Coverage Optimization for Community Electrical Fire Monitoring
}

\author{
Tingting $\mathrm{Li}^{1}, \mathrm{Bo} \mathrm{Li}^{2,}$, Dechang Huang ${ }^{2}$ \\ ${ }^{1}$ East China Jiaotong University, Software School, Nanchang, Jiangxi, China \\ ${ }^{2}$ East China Jiaotong University, Information Engineering School, Nanchang, Jiangxi, China
}

Keywords: Community electrical fire, Fire monitoring, Monitoring system design, Network coverage optimization.

Abstract: The community's convenience services and security protection are gradually becoming more technological and intelligent. The ecological transformation of the community should be driven by informationization. By developing innovative construction models and using advanced technologies to change the consumption patterns, decision-making methods, management methods and security measures of traditional communities. ZigBee technology is a hot-port technology in the field of wireless sensor technology research in recent years. In life, ZigBee technology is often used in production environment monitoring, industrial monitoring and so on. Therefore, by analyzing the technical characteristics of ZigBee technology, the ZigBee module is used as the sensing node of the wireless sensor network, and the wireless security network is established by using the characteristics of low power consumption and easy networking of the ZigBee node. This paper focuses on the design of electrical fire monitoring systems and network coverage optimization, in order to provide design experience for the automatic and efficient identification of electrical fires.

\section{Introduction}

With the acceleration of urban construction, rapid economic development and the continuous prosperity of industry, various electrification products are widely used in our daily lives. Since the actual installation and wiring needs to cooperate with the construction of the building, there are many factors to be considered in the event of equipment failure for secondary maintenance and disassembly, which undoubtedly increases the installation cost. Therefore, many electrical equipments are subject to irregular installation or unreasonable wiring, plus management and use of omissions, will create many uncertain security issues. Sometimes it will inevitably cause some electrical accidents and even cause electrical fires. As an important part of the town, the community is in close contact with daily life. Once such an accident occurs, it is usually accompanied by huge economic losses and casualties, and the consequences are unimaginable. Therefore, this paper mainly analyzes the technical characteristics of ZigBee technology, uses ZigBee module as the sensing node of wireless sensor network, and uses ZigBee node to establish wireless security network with low power consumption and easy networking. Focus on the design of electrical fire monitoring system and network coverage optimization, in order to provide design experience for the automatic and 
efficient identification of electrical fires, and share with the majority of peers[1].

(1) Main characteristics and identification of community electrical fires. Electrical fires mainly exhibit several aspects as shown in Table 1.

Table 1 Characteristics of electrical fires

\begin{tabular}{c|c}
\hline Short burst time & $\begin{array}{c}\text { Electrical fire failures often break out in an instant and are often not } \\
\text { easily noticeable. }\end{array}$ \\
$\begin{array}{c}\text { The scope of the } \\
\text { explosion has } \\
\text { expanded rapidly }\end{array}$ & $\begin{array}{c}\text { Once an electrical fire breaks out, due to the influence of adjacent } \\
\text { electrical components and lines, the scope of the explosion is rapidly } \\
\text { expanding, which brings certain difficulties to the prevention and } \\
\text { suppression of electrical fires. }\end{array}$ \\
Long latency & $\begin{array}{c}\text { The fault characteristics of electrical fires are weaker than those of other } \\
\text { electrical faults, and it is generally not easy to capture the fault } \\
\text { characteristics of electrical fires. }\end{array}$ \\
\hline
\end{tabular}

Although the fault characteristics of electrical fires are not easily captured, there are still some fault features that can be captured and identified, and this is the main identification technology basis for current electrical fire monitoring. If the residual current is abrupt, that is, according to the statistics of electrical fire accidents, when an electrical fire occurs, there will be a large residual current in the circuit before the occurrence, and this residual current is the culprit causing the electrical fire. Another example is the sudden change in temperature, that is, when there is an electrical fire in the circuit, the temperature in the circuit rises sharply before the fire occurs[2].The use of high-precision temperature sensors to capture sudden changes in temperature in the circuit, so as to take appropriate countermeasures and measures to reduce or reduce or even completely extinguish the electrical fire.

(2) Electrical fire monitoring system. The electrical fire monitoring system consists of an electrical fire monitoring host, a temperature-measuring electrical fire detector installed on the monitoring object, a residual current electrical fire detector, a wireless data concentrator, and a field bus. An electrical fire monitoring host is installed in each building of the community. The electrical fire monitoring system of each building is a relatively independent system. In the event of a failure of the backbone transmission network, the electrical fire monitoring system of each building can still work normally. The structure of the electrical fire monitoring system is shown in Figure 1.

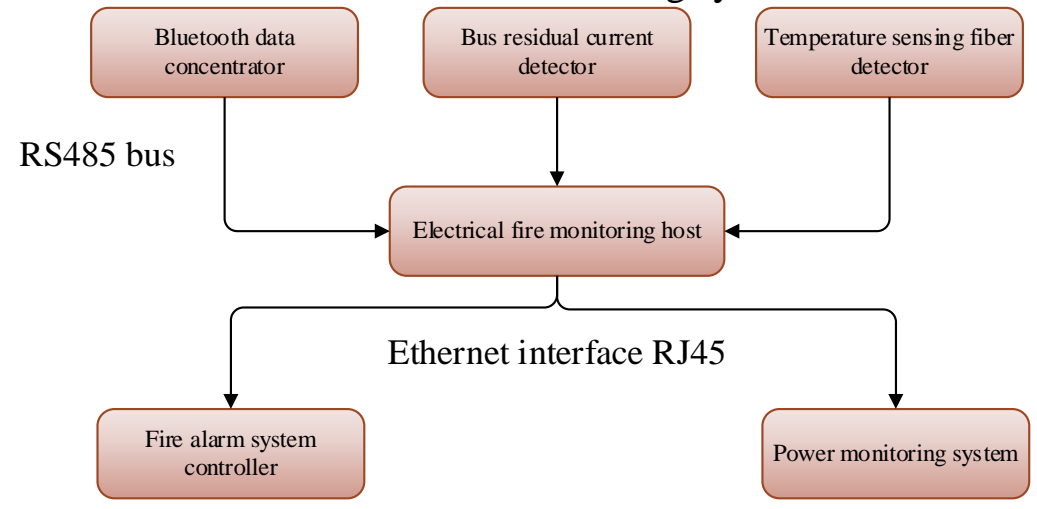

Figure 1 Structure diagram of electrical fire monitoring system

\section{Introduction to wireless sensor networks and ZigBee related technologies}

With the development of smart cities, smart communities and smart homes in China, wireless sensing technology will provide important technical support for information acquisition, signal transmission and network coverage. The communities in which we live are characterized by a wide 
range of features, a large number of people, and a large amount of data. The advantages of wireless sensor network networking, large coverage area, and convenient access to information can meet people's needs. The characteristics of the wireless sensor network mainly include large-scale, self-organizing, multi-hop routing/dynamic, and data-based networks.

As a new type of sensing technology, ZigBee technology has its unique advancement. These technical characteristics determine that it is more suitable for the construction of wireless sensor networks in the smart community. ZigBee technology features low power consumption, low cost, short delay, large network capacity, high reliability, high security and high openness. Because ZigBee technology has many advantages that are unmatched by other information technologies, this technology has a larger application range and a broader application space. In the future, people will pay more attention to the application design and technology marketing of ZigBee technology. The foundation of ZigBee's protocol stack is still the structure of OSI. The PHY layer and MAC layer of ZigBee are defined on the international common standard protocol[3]. The ZigBee Alliance defines its APP layer, security layer and NWK layer. Among them, the security layer exists in each layer of the protocol, it is not a layer in which the entity exists, but is a general term after the security function of each layer is attributed. Combine the various layers of protocols and implement the functions of the protocol through functions. As shown in Figure 2.

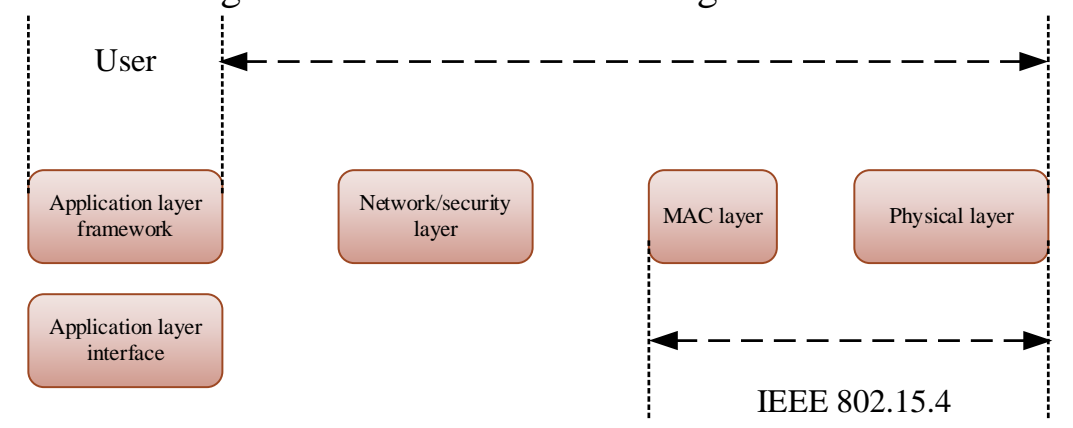

Figure 2 Model diagram of the ZigBee protocol stack

\section{Community fire prevention and control system design and monitoring}

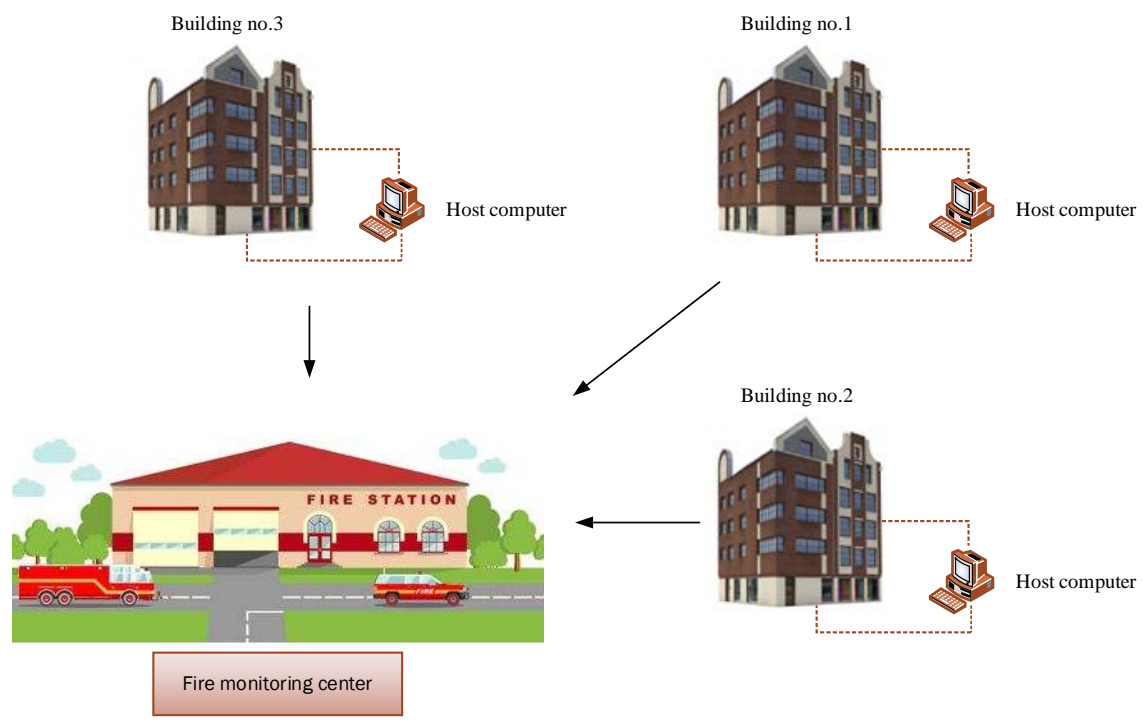

Figure 3 Community real monitoring scenario

(1) Overview of the overall design. This paper mainly designs the fire protection subsystem in 
the intelligent security in the community. Here, the ZigBee module needs to collect the concentration information and temperature information, and alarm PUSl by detecting the abnormality early. Once the fire occurs, the firefighters on the spot can be located. The firefighters wear the equipment with the ZigBee module. Before entering the fire, they can be added to the site network in time. Therefore, it is convenient to conduct the positioning of personnel on site or remotely, that is, it is convenient for personnel assistance, and it is also convenient to dispatch on-site scheduling. The system needs to monitor all the electrical equipment of a community building group[4]. The approximate geographical distribution of the community building group and the fire monitoring center in the actual monitoring scenario is shown in Figure 3.

It can be seen from Figure 3 that the community buildings are about a few hundred meters apart from each other. The fire control system control center needs to collect all building monitoring information into the fire control center for unified management. The structure of the internal monitoring area of a single building is roughly as shown in Figure 4.

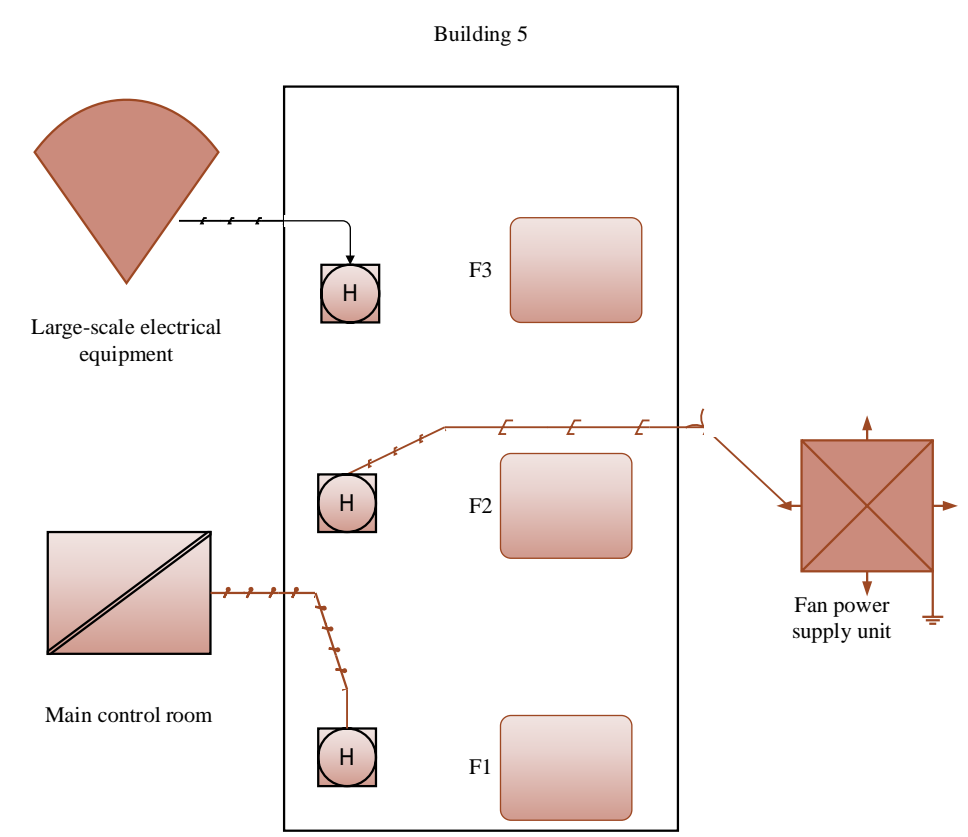

Figure 4 Building distribution facilities are generally distributed

The system requires 24/7 networked monitoring of all surveillance areas. Overcoming the inconvenience of on-site inspection of traditional electrical fire monitoring systems, the network of the entire community building group can be grasped at any time through the network. Realize the convenient management of real-time monitoring, everywhere viewing, and timely warning. The system needs to achieve more intelligent monitoring. Combined with Internet of Things technology, computer network technology and visualization technology, it provides intelligent services for comprehensive monitoring of monitoring data, diversified presentation of data forms, and full control of equipment operation status for the monitoring and management of electrical fires in this community. In order to improve the real-time nature of fire prevention, the environmental monitoring system designed in this paper mainly collects temperature and concentration information[5]. In order to improve the fire field dispatching ability, the article also designed on-site positioning capability.

(2) System software design. The software part of the system, including the operating interface of the host computer, ie the PC, the design of the database, data analysis and processing. And the design of the lower part machine, that is, the embedded part coordinator, the routing device, the terminal node, and the design of the algorithm part. Including information collection, detection algorithm 
design and node positioning algorithm design, as shown in Figure 5.

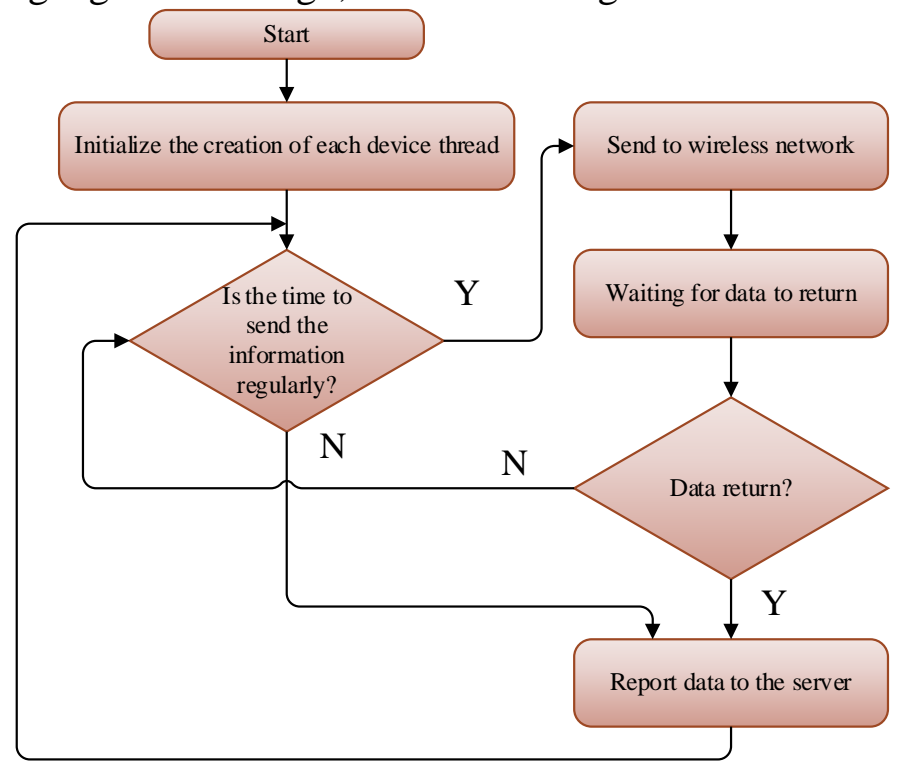

Figure 5 Data acquistion menagement flow chat

\section{Network over-coverage optimization scheme for community electric fire monitoring based on energy balance}

Due to the existence of network holes, the network is not complete and cannot comprehensively monitor the target area. This chapter proposes a network over-coverage optimization scheme based on energy balance for the coverage hole problem generated by random deployment of static wireless sensor networks.

(1) Network model creation combined with energy balance. In some specific situations, such as disaster scenes, because the staff is difficult to get close, it is necessary to use the sensor network to find the ability to cover the blind spot. In the first step, the network state model is created by the cooperative detection probability and the energy distribution of the node, and then the position information of the coverage hole is obtained by using the set network state threshold, and then the edge of the cavity is determined. Secondly, the triangulation algorithm is used to divide the cavity area to obtain the best node re-deployment location, thereby improving the comprehensive coverage of the network and achieving better service quality.

(2) Research on coverage hole detection based on energy balance. In the first step, the corresponding network model is created by the cooperative detection probability of the node and the energy distribution, and then the position information of the coverage hole is obtained by using the threshold, and then the edge of the cavity is obtained. Secondly, the relevant triangular algorithm is used to divide the cavity area to get the best node position, which improves the comprehensive coverage and achieves better service quality. The specific concept of the model is shown in Equation 1.

$$
F_{p}=\sqrt{D_{p}(s) \times\left(1+\sum_{i=1}^{N}\left(1-E_{i}^{D M S A}\right)\right)}
$$

Among them, $\sum_{i=1}^{N}\left(1-E_{i}^{D M S A}\right)$ represents the actual energy consumption of point p. 
$D_{p}$ represents the cooperative detection probability corresponding to the point p.

$F_{p}$ represents the specific network state value in the process of calculating the cooperative monitoring probability and energy consumption[6].

(3) Research on network coverage hole detection. After the network realizes the initial random deployment of nodes, in the integrated two-dimensional monitoring space, the actual detection probability of each node is closer to the exponential mode. In the case where each node works together, the network state value of all nodes is calculated by the network state model. When the network status value is zero, the node cannot perform effective detection and is regarded as covering the hole. Some points keep the parameters low in terms of network state values but not zero, that is, there is a probability of being detected, but the actual probability is relatively low. In order to ensure that the target can be effectively monitored, the paper defines the corresponding threshold (in the specific application process, the actual coverage rate is required to set up).That is, a point where the state value is greater than the threshold value enables efficient detection, and a point below the threshold value cannot be detected.

\section{Conclusion}

The monitoring of electrical fires requires the cooperation of the entire power system to truly realize the electrical fire prevention of the whole circuit, and cannot rely solely on the power monitoring department. Achieve all-round visual monitoring, prediction, alarm, analysis and disposal of electrical fires, the realization of electrical fire monitoring information can effectively expand the application areas and monitoring scope of electrical fire monitoring, greatly improve the level of prevention and control of electrical fires and technical level, and is of great significance for protecting people's lives and property and building a harmonious society.

\section{Acknowledgment}

This work was supported by Natural Science Foundation of China (No. 61863013), Key R \& D projects of Jiangxi science and Technology Department of China (No. 20161BBE50091), Science and Technology Foundation of Jiangxi Educational Committee of China (No. 150529), and East China Jiaotong University School Foundation Fund "Research on Urban Fire Monitoring System Based on IoT Collaboration Perception" (15RJ01).

\section{References}

[1] Khedr, Ahmed M. New Localization Technique for Mobile Wireless Sensor Networks Using Sectorized Antenna[J]. International Journal of Communications, Network and System Sciences,2015,8(9). 329-341.

[2] Hua L, Shao G. The progress of operational forest fire monitoring with infrared remote sensing[J]. Journal of forestry research, 2017, 28(2): 215-229.

[3] Shvetsov E G, Kukavskaya E A, Buryak L V. Satellite monitoring of the state of forest vegetation after fire impacts in the Zabaikal region[J]. Contemporary Problems of Ecology, 2016, 9(6): 702-710.

[4] Fatih Senel,Kemal Akkaya,Melike Erol-Kantarci,Turgay Yilmaz. Self-deployment of mobile underwater acoustic sensor networks for maximized coverage and guaranteed connectivity [J]. Ad Hoc Networks,2015,34. 170-183.

[5]Carolina Filizzola,Rosita Corrado,Francesco Marchese,Giuseppe Mazzeo,Rossana Paciello,Nicola Pergola,Valerio Tramutoli. RST-FIRES, an exportable algorithm for early-fire detection and monitoring: description, implementation, and field validation in the case of the MSG-SEVIRI sensor[J]. Remote Sensing of Environment,2016,186. 196-216

[6] Radu Stoleru,Haijie Wu,Harsha Chenji. Secure neighbor discovery and wormhole localization in mobile ad hoc networks[J]. Ad Hoc Networks,2012,10(7). 1179-1190. 